\title{
A exposição Internacional de 1862 em Londres e a origem das megaexposições de arte contemporânea
}

Vinicius Spricigo ensina curadoria e história das exposições no Departamento de História da Arte da UNIFESP. Foi Curador associado ao Fórum Permanente: Museus de Arte; entre o público e o privado (2006-2008) e à Associação Cultural Videobrasil (para a realização da exposição Geopoéticas do artista Isaac Julien no SESC Pompéia, 2012). Realizou pesquisa de pós-doutorado junto ao Research Centre for Transnational Art Identity and Nation da University of the Arts London (2019-2020) e contribuiu, entre outros textos publicados, para os livros The Biennial Reader e German Art in São Paulo, ambos pela Hatje Cantz. <vinicius.spricigo@unifesp.br> ORCID: 0000-0003-3320-1805
Resumo $O$ presente artigo trata da origem das exposições internacionais de arte no século XIX, sobretudo as exposições de Londres de 1851 e 1862, enquanto modelo para as bienais contemporâneas. Nele é apresentada uma revisão historiográfica sobre o tema, abrangendo também outros modos de exposição criados no período, como os museus de arte e as exposições sazonais da academia. 0 trabalho trata ainda da questão das representações nacionais nesse âmbito.

Palavras chave Exposições Internacionais , Londres, Representações nacionais, Curadoria. 


\section{London's International Exhibition of 1862 and the origin of large scale exhibitions of Contemporary Art}

Abstract This paper analyses the origin of international exhibitions in the nineteenth century as a model for contemporary biennials, with emphasis on London exhibitions of 1851 and 1862. It presents a bibliography review about the subject, comprising other modes of exhibition created in that period, such as museums and periodical exhibitions held by the art academy. The article approaches the question of national representation in this realm.

Keywords International Exhibitions, London, National Representations, Curating.

La exposición internacional de 1862 en Londres y el origen de las mega-exposiciones de arte contemporáneo

Resumen Este artículo aborda el origen de las exposiciones internacionales de arte en el siglo XIX, especialmente las exposiciones de Londres de 1851 y 1862, como modelo para las bienales contemporáneas. Presenta una revisión historiográfica sobre el tema, cubriendo también otras modalidades expositivas creadas en la época, como los museos de arte y las exposiciones estacionales de la academia. El trabajo también aborda el tema de las representaciones nacionales en este contexto.

Palabras clave Exposiciones Internacionales, Londres, Representaciones Nacionales, Curaduría. 


\section{Introdução}

Gostaria de iniciar com algumas considerações introdutórias sobre a metodologia empregada na pesquisa realizada durante um estágio de pós-doutorado no Research Centre for Transnational Art Identity and Nation da University of the Arts London, no segundo semestre de 2019, com o apoio da Fundação de Amparo à pesquisa no estado de São Paulo (FAPESP). Em primeiro lugar, faço uma consideração acerca da nomenclatura aqui utilizada. No projeto inicial, ponto de partida para a pesquisa, foi empregado o termo megaexposições 'periódicas' de arte contemporânea com referência aos estudos de exposições bienais dentre os quais ela se insere. No entanto, quando aplicado às megaexposições do século XIX, o termo apresenta algumas inconsistências. Embora tenham sido realizadas com alguma periodicidade, sobretudo as expositions universelles de Paris (1855, 1867, 1878, 1889, 1900 e 1937), as exposições de Londres, por exemplo, foram interrompidas na sua segunda edição. Diferentemente do modelo surgido com a Biennale di Venezia (1895), as megaexposições do século XIX não apresentavam um regularidade ou periodicidade definida, mesmo que as constantes repetições possam sugerir tal aspecto. Não se trata de um detalhe menor. Pelo contrário, os estudos sobre as bienais pregam que as megaexposições periódicas de arte contemporânea seguiram o modelo novecentista (Filipovic; Van Hal; Øvstebø, 2010). Contudo, se a organização das exposições tendo como formato as representações nacionais demonstra uma transição do período moderno ao contemporâneo, a recorrência de tais exposições no século XIX, a meu ver, não deveria ser confundida com uma serialidade. A depender do seu sucesso ou de condições financeiras e políticas favoráveis, novas edições de uma determinada exposição poderiam ser novamente realizadas ou não. Investigações futuras podem aprofundar essa discussão, abordando a forma como a Biennale di Venezia inaugura um novo modelo de megaexposições que se repetem numa série regular sem interrupções. Por hora, no presente artigo, passo a utilizar o termo (mega)exposições internacionais para designar os eventos ocorridos no século XIX, e (mega)exposições periódicas quando me refiro às bienais criadas segundo o modelo veneziano.

Da mesma forma, a ideia da origem das megaexposições periódicas de arte contemporânea em meados do século XIX pode ser repensada, ao menos quando situamos como marco inaugural a Great Exhibition of the Works of All Nations, realizada em 1851, no Crystal Palace projetado por Joseph Paxton. A bibliografia sobre as megaexposições internacionais do século XIX destacam o impacto que a construção de Paxton teve sobre o público, criando aliás uma grande expectativa com relação aos planos de uma exposição subsequente em Londres, que seria frustrada com a construção de um novo edifício em 1862 (DISHON, 2017: 70). Entretanto, a importância atribuída ao Palácio de Cristal não se resume ao seu aspecto espetacular. Segundo Donald Preziosi, o Palácio de Cristal, com sua transparência, seria 
um 'véu cristalino' que cobre e ao mesmo tempo revela a posição de centralidade epistemológica assumida pela história da $\operatorname{arte}^{1}$ como o 'cérebro do corpo do planeta' (PREZIOSI, 2003). A Grande Exposição de 1851, assim como a história da arte, permitem não somente reunir objetos dos quatro cantos do mundo, mas também organizá-los hierarquicamente e atribuir significados a partir dessa lógica de organização, segundo Preziosi (2003). Adotado com marco teórico para o estudo das bienais (Filipovic; Van Hal; Øvstebø, 2010), Preziosi aponta para a origem das exposições internacionais na Grande Exposição de 1851. Tamanha importância dada ao Palácio de Cristal enquanto marco inaugural de uma história das megaexposições do século XIX faz com que a exposição tenha obscurecido a sua sucessora, como sugere John Davis:

While the Exhibition of 1851 is known as 'Great', that of 1862 is not given any soubriquet. The Great Exhibition of 1851 is a landmark for historians of Victorian Britain, widely viewed as the highpoint, totem, or epitome of the age, and linked specifically to the emergence of Britain from a period of unrest into one of economic growth, confidence and equipoise. That of 1862, meanwhile, is rarely mentioned in general histories, scarcely known about in detail and frequently lost in the usual chronological accounts of the period. If it is remarked on at all, it is usually as a sort of ugly sister to the glorious event eleven years previously in Hyde Park: a warped reflection of the Crystal Palace, of which Thomas Prasch asks 'Can a palace of glass cast a shadow?' (DAVIS, 1999: 10)

Apesar de ter sido encoberta pelas sombras do Palácio de Cristal, a exposição de 1862 nos interessa sobremaneira. Um aspecto que parece ter passado desapercebido para Davis e outros autores que tratam do tema foi a atribuição à segunda megaexposição de Londres da alcunha de 'Internacional'. Apesar de analisarem a internacionalização das exposições do ponto de vista do imperialismo britânico da Era Vitoriana, os autores dão pouco atenção ao fato da exposição de 1862 ter sido a primeira a adotar essa nomenclatura, ao substituir o adjetivo 'grande' por 'internacional'. Ademais, não há menções para as possíveis distinções do termo 'universal' empregado pelos franceses no mesmo período. Paul Young (2009), único autor a problematizar essa questão ao vincular uma visão político-econômica da globalização ao fenômeno das exposições, acaba recaindo na hipótese mais aceita da associação da Grande Exposição com a expansão capitalista e a industrialização do século XIX. Aliás, não foi somente a grandiosidade da exposição de 1851 a responsável pelo seu tremendo sucesso a ponto de ser considerada como a fundadora de um modelo de megaexposições. A inclusão de Works of All Nations fez com ela tenha superado também neste aspecto as suas antecessoras, as mostra nacionais realizadas repetidamente em Paris em 1802, 1806, 1819, 1823, 1827, 1834, 1839, 1844 and 1849. Ao romper 
com o localismo dessas precursoras, a Grande Exposição de 1851 teria inaugurado uma nova era de expansão comercial e softpower atrelados ao entretenimento do público. Talvez a mudança na nomenclatura em 1862 tenha sido resposta à Exposition Universelle de 1855, a mesma que realizou uma grande transformação no modelo das megaexposições ao incluir a apresentação de obras de arte ao lado dos avanços tecnológicos e dos resultados da sua aplicação na fabricação de produtos comerciais. Além de ter sido aclamada pelo público, a galeria de pinturas da Exposição Internacional de 1862 foi responsável por uma ampliação significativa no campo conceitual das exposições de Londres, bem como com relação à primeira tentativa francesa de apresentar a arte contemporânea em uma exposição internacional em 1855 (INTERNATIONAL EXHIBITION, 1862a: 50). Em comparação com essa última, a exposição de 1862 não se resumiu a incluir as artes plásticas em suas galerias, mas tratou também de apresentar uma delimitação temporal que incluísse obras de um passado recente. Notavelmente, ao invés de destacar importantes nomes contemporâneos como fizeram os franceses com Eugene Delacroix, Jean-Auguste-Dominique Ingres e Gustave Courbet em 1855, a representação inglesa apresentava a formação de uma escola nacional com William Hogarth, Joshua Reynolds, Thomas Gainsborough e William Turner (BRYANT, 2014: 58-81). Essa disputa entre Londres e Paris na realização de megaexposições atraindo milhões de visitantes marca o contexto de rivalidade entre os dois maiores impérios do século XIX e, nesse aspecto, a exposição de 1862 parece ter tido um papel mais importante para a historicização da arte moderna do que as suas precursoras, mesmo sem ter recebido a mesma atenção dos historiadores.

Ao abordar as megaexposições dando ênfase ao modo como elas articularam o binômio nacional-internacional, o presente estudo tem o intuito de avançar no debate atual para além dos dois aspectos (sazonalidade e espetacularidade) que marcam os eixos dos estudos até agora citados. Para nós, compreender as exposições como uma 'arena pacífica' (locais de disputa regulamentada e premiações) para o exercício do soft power pelas nações hegemônicas, nas quais as diferenças são definidas a partir de uma concepção internacionalista (ou universalista), é uma invenção moderna que persiste até os dias de hoje, como 'a dream from which we have yet to awaken' (PREZIOSI, 2008: 97). Diferentemente de uma Babel, como sugere um texto de época (INTERNATIONAL EXHIBITION, 1862b: 353), onde cada indivíduo fala sua própria língua, no espaço das exposições internacionais fala-se a língua colonial do internacionalismo ou do globalismo (avant la lettre). Nesse sentido, a nossa reflexão sobre a origem do internacionalismo em exposições do século XIX, pode contribuir para o estudo de exposições bienais e outras megaexposições de arte contemporânea. 


\section{Revisão historiográfica}

A pesquisa no exterior permitiu acesso à livros, periódicos e teses publicados no Reino Unido, ou seja, à uma bibliografia em língua inglesa que fundamentou a análise da Exposição Internacional de 1862. Confirmou-se a hipótese apresentada anteriormente de que a exposição de 1862 foi praticamente esquecida. Mesmo Rezende (2010), pesquisadora brasileira que cursou história do design no Royal College of Art, um curso promovido em parceria com o Victoria \& Albert Museum, instituição que abriga a documentação referente às megaexposições realizadas em Londres, em 1851 e 1862, deu pouca atenção para essa última. ${ }^{2}$ Apesar de sua importância para o estudo das representações brasileiras em megaexposições no século XIX e início do século $\mathrm{XX}$, sobretudo para o campo do design, do ponto de vista metodológico, a fundamentação teórica empregada por Rezende se alinha conceitualmente à obra Ephemeral Vistas, ${ }^{3}$ adotando uma narrativa histórica que se restringe à análise dos objetivos imperialistas das exposições internacionais, um problema aliás já apontado por Davis com relação à Greenhalgh (1999: XV). Outro problema apontado por Davis, entre o número crescente de publicações que surge sobre o tema a partir dos anos 1990, é o tom celebratório e a tendência a glorificar a Grande Exposição de 1851, visto em obras como 'The Great Exhibitions: 150 years', originalmente publicada por John Allwood, em 1977, quando o assunto ainda era negligenciado por muitos autores e pesquisadores. Outro exemplo de reducionismo é a obra de Nikolaus Pevsner, 'High Victorian Design', publicado na ocasião da realização do Festival of Britain em 1951. A crítica do autor à produção industrial apresentada na Grande Exposição de 1851 estava fundamentada somente numa seleção de produtos exibidos e artigos publicados no 'The Art Journal', sem dar a devida atenção aos aspectos mais amplos que envolveram o desenvolvimento do design britânico em meados do século XIX. A atitude de Pevsner com relação ao design vitoriano, afirma Davis, 'passed on to a later generation: his standards [damning Victorian aesthetics] became the new aesthetic orthodoxy, possibly with disastrous results for the treatment of Victorian heritage' (1999: XV).

Naquilo que concerne a nossa pesquisa, tantos os trabalhos seminais de Pevsner (1951) e Allwood (2001), quanto as abordagens históricas de Greenhalgh (1988) e Davis (1999), assumem sem contestações a Grande Exposição de 1851 como uma marco inaugural, com breves menções à eventos precedentes, como as feiras medievais, as exposições industriais da França pós-revolucionária, bem como as exposições promovidas pela Society of Arts e as exposições de verão da Royal Academy, centrais para se compreender a emergência de uma noção moderna de exposição no século XIX. Aqui também o livro de Richard Altick (1978), 'The Shows of London', deve ser mencionado, por apontar para uma grande variedade de 'entretenimentos' públicos como gabinetes de curiosidade, museus de cera, exibições de engenhocas, panoramas e dioramas, freak e folk shows, jardins zoológicos, que vão da "Middle Ages, with "scientific" and "historical" rarities [as objects of curiosity]' até a 'decade following that exhilarating apotheosis of the London exhibition, the Chrystal Palace of 1851.' (Altick, 1978: 3) 


\section{Origem das exposições e dos museus}

$\mathrm{Na}$ medida em que compreendemos que a origem das exposições internacionais está inserida nesse contexto mais amplo, a Grande Exposição de 1851 passa a ser visto como mais um evento importante numa sucessão de outras realizações iniciadas na primeira metade do século XIX, sobretudo na França pós-revolucionária (GREENHALG 1988: 3-8). Não por acaso, o surrealista George Bataille assinalou as ligações entre a Revolução Francesa e o surgimento de exposições públicas, espetáculos e museus. 'The origin of the modern museum [...] is linked to the development of the guillotine', escreveu Bataille (1986: 24). Importante ressaltar ainda uma sincronia no surgimento de exposições e de museus modernos, conforme assinala Tony Bennett (1995). O autor tem como ponto de partida a oposição que Michel Foucault traça entre os museus, enquanto um espaço heterotópico que permite reunir todas as temporalidades e especialidades no seu interior, e as feiras populares, com suas localidades descentralizadas nos arredores das cidades e seu apelo à curiosidade e ao entretenimento. No entanto, Bennett ressalta que os parques de diversões, que surgem nos Estados Unidos a partir das World Fairs, estão situados em uma zona nebulosa onde essa distinção e oposição não estão bem definidas. Além disso, esses 'outros espaços', assim como as exposições internacionais, são organizados a partir da mesma lógica dos museus, segundo ele (BENNETT 1995). O livro sobre 'The Birth of the Museum' busca assim compreender a lógica de organização desses espaços a partir de uma fundamentação teórica baseada certamente em Foucault, mas também em Gramsci, Habermas e autores da área da museologia. No âmbito desta pesquisa, interessa sobremaneira alguns aspectos assinalados por Bennett, bem como por autores como Carol Duncan (2004), a saber: o papel do colecionismo na formação dos museus modernos; a relação entre a formação de museus e estados nacionais no século XIX; o papel dos museus na definição e divulgação da noção de cidadania e dos comportamentos necessários para o convívio social dentro da nova ordem política estabelecida após o fim dos absolutismos. Para dar um exemplo, poderíamos comparar o studiolo de Francisco I de Medici no Palazzo Vecchio em Florença e os displays das exposições universais de Paris. No primeiro, o acesso privado é restrito ao próprio soberano. Sua escala é limitada a um pequeno estúdio no qual o próprio 'príncipe' assume o centro da representação. Mais tarde quando as coleções reais são abertas ao público - ainda no sentido restrito àqueles que podiam adentrar as Galerias dos Ofícios a partir de 1765 , lembrando que elas se tornaram um museu público no sentido moderno somente em 1865, após a unificação italiana - o governante continua sendo o centro das representações mesmo que agora o reconhecimento do espectador seja requerido. Nas exposições universais essa lógica é transformada completamente. No centro da representação está o progresso da sociedade moderna, no limite o capital colocado no centro do prédio construído para a Exposition Universelle de 1867 em Paris. Além disso, o acesso público é massivo e, portanto, o seu fluxo deve ser organizado e controlado. Por esse motivo, são 
construídas obras como o Palácio de Cristal e as salas e aposentos dos antigos palácios como o Louvre foram transformadas em galerias de exposição quando esses foram convertidos em museus. Da mesma forma, a organização das obras que antes representava a centralidade do rei como soberano, passa a ser estabelecida a partir de um discurso sobre a nação, bem como as obras e artistas são organizados por escolas nacionais, e sua apresentação está fundamentada no discurso estético da história da arte.

ta Duncan:

O museu do Louvre foi 'the prototypical public art museum', argumen-

It first offered the civic ritual that other nations would emulate. [...] The Louvre was not the first royal collection to be turned into a public art museum, but its transformation was the most politically significant and influential. In 1793 the French Revolutionary government, seizing an opportunity to dramatize the creation of the new Republican state, nationalized the king's art collection and declared the Louvre a public institution. The Louvre, once the palace of Kings, was now reorganized as a museum for the people, to be open to everyone free of charge. It thus became a lucid symbol of the fall of the old Regime and the rise of a new order. (DUNCAN 2004: 250-251)

Com o objetivo de alcançar seu novo objetivo público, o museu moderno precisou reorganizar a sua de forma organizar as coleções da realeza seguindo inicialmente uma nova forma de 'bourgeois connoisseurship'. De acordo com Duncan (2004: 254), após a Revolução Francesa, esse 'gentlemanly type of installation' de coleções privadas e principescas deu lugar aos novos 'art-historical arrangements' dos museus modernos:

Historians of museums often see the new art-historical hang as the triumph of an advanced, Enlightenment thinking that sought to replace earlier systems of classification with a more rational one. To be sure, the new construct was more in keeping with Enlightenment rationality. But more significant to the concerns of this study was its ideological usefulness to emerging bourgeois states, all of which, in the course of nineteenth century, adopted it for their public art museums. Although still pitched to an educated elite and still built up on a universal and international standard, the new system, by giving special emphasis to the 'genius' of national schools, could both acknowledge and promote the growth of state power and national identity. (DUNCAN 2004: 255)

Desde o início, as galerias dos museus foram organizadas por escolas nacionais. No caso do Louvre, após a sua reabertura em como Musée 
Napoléon sob a direção de Vivant Denon (DUNCAN 2004: 256). Embora essa narrativa histórica possa ser generalizada para todos os países da Europa, bem como para aqueles das Américas que constituíram seus museus nacionais no modelo europeu, há distinções claras quando analisamos o caso britânico neste estudo. Retornando à afirmação de Bataille sobre a origem dos museus modernos, pode-se igualmente sublinhar o contraste com os empreendimentos privados da Grã Bretanha, quando ele aponta que: 'Nevertheless, the collection of the Ashmolean Museum in Oxford, founded at the end of the seventeenth century, was already a public one, belonging to the university' (Baitaille 1986: 24). Carecendo de uma coleção real que pudesse ser transformada em patrimônio nacional (por razões históricas, a coleção constituída por Carlos I na primeira metade do século XVII foi desfeita após a sua deposição e execução e os museus britânicos foram criados a partir de coleções privadas), as instituições britânicas respondiam ao gosto dos colecionadores e não traziam consigo, necessariamente, a mesma ideologia que na França fundamentaria os discursos nacionalistas e identitários. Além disso, a criação de uma galeria nacional no Reino Unido aconteceu somente 1824, tendo a coleção privada de John Julius Angerstein como seu núcleo central, apesar de muitas dessas coleções já estarem disponíveis à visitação pública mediante o pagamento de uma entrada, como era o caso da coleção de 'raridades' da família Tradescant recebida por Elias Ashmole e posteriormente doada à Universidade de Oxford em 1677. Nesse sentido, acrescenta Duncan:

\footnotetext{
Art galleries signified social distinction precisely because they were seen as more than simply signs of wealthy and power. Art was understood to be a source of valuable moral and spiritual experience. In this sense, it was cultural property, something to be shared by a whole community. Eighteenth-century Englishmen as well as Frenchmen had the idea that an art collection could belong to a nation, however they understood that term. The French pamphleteers who called for the nationalization of the royal collection and the creation of a national art museum had British counterparts who criticized rich collectors for excluding from their galleries a large public, especially artists and writers. (DUNCAN 2004: p.265-266)
}

Ao lado da criação dos museus, um dos principais instrumentos para permitir o acesso do público à arte, antes um privilégio de colecionadores e nobres, foram as exposições de arte. No Reino Unido, instituições como a Society of Arts, criada em 1754, desempenharam um papel importante na realização de exposições - notavelmente a exposição anual da academia, sobretudo pelo fato dela ter contado inicialmente com o apoio da sociedade pela seção de uma sala para exposições anuais de artistas que a precederam - e na constituição de galerias de arte abertas ao público, bem como foi a 
Society of Arts a principal promotora das duas edições da megaexposições de Londres. Segundo Sidney Hutchison, a sociedade realizou em 1759, uma exposição anual de arte contemporânea que 'may be looked upon as a father figure of the Royal Academy', fundada em 1768, e 'the forerunner of the Summer Exhibitions held annually from 1769' (Hutchison 1986: 17). Para Davis (1999: 4), 'these art exhibitions were organised by a group of artists that breakup with the Society of Arts and held independently until 1783'.

O papel desempenhado pela Society of Arts, relatado no anuário da Exposição Internacional de 1862, incluiu ainda colecionar matérias primas e produtos manufaturados e organizar exposições anuais de produtos manufaturados e tecnologias industriais. $O$ que parece estar em questão em meados do século XIX é uma evidente divisão entre exposições industriais organizadas pela Society of Arts e exposições de arte organizadas por artistas dissidentes que levaram à criação da Royal Academy, a qual em 1851 estava situada em Trafalgar Square na região central de Londres juntamente com a National Gallery, dividindo assim a atenção do público naquele verão com a Grande Exposição situada no Hyde Park. Por fim, do ponto de vista da sustentabilidade das exposições, a diferença com relação aos eventos ocorridos na França é significativa, uma vez que embora tenham o apoio do governo elas não dependem exclusivamente dele para a sua realização. Nas megaexposições de Londres, o envolvimento do empresariado, da elite econômica e a cobrança pelos ingressos eram fundamentais para a sua realização.

\section{Internacionalização das exposições}

Se em muitos aspectos 'the activities of fairs, museums and exhibitions interacted with one another' (Bennet 1985: 5), no entanto, o marco inaugural para uma serie de megaexposições foi a internacionalização de um padrão estabelecido pelas exposições industriais francesas da primeira metade do século XIX. Em 1849, Henry Cole, servidor do Public Record Office que organizou a primeira Annual Exhibition of Select Specimens of British Arts and Manufactures em 1847, visitou a French National Exhibition e no seu retorno ele 'had the opportunity to discuss with Prince Albert the possibility of making the London exhibition an international one' (Young 2009: 17). No entanto, como assinala Davis, 'one cannot pinpoint any particular person to whom the idea of throwing open the doors of the exhibition for foreign country can be attributed'. (Davis 1999: 10) Apesar da ênfase que a bibliografia dá ao papel desempenhado por Cole e pelo Príncipe Albert como os principais organizadores da Great Exhibition, é importante compreender nesse contexto a confiança dos empreendedores britânicos em seus produtos, decisivos para a decisão de organizar uma primeira exposição internacional, afirma Davis: 
By 1850 [...], when the largest exhibition ever held in Britain was going through its initial planning phase, it was decided, for the first time anywhere, to invite all nations of the world to participate. The raisin d'être of English exhibitions had thus gone through an abrupt change. Whilst in London English industry was still considered to lead the world, it was also recognised that competition was growing and that the only way to defeat it was to out-sell it. To invite all nations of the world to take part in 'the friendly competition' of an international exhibition was to create a potential market expansion abroad. One of the main motives behind the international character of the Great Exhibition of 1851 therefore was economic; Britain wanted more market, and was confident when foreigner came he would by British goods. (DAVIS 1999: 10)

O escopo internacional - bem como outras decisões como a divisão das galerias em quatro núcleos: matérias primas, maquinário, produtos manufaturados e escultura e artes plásticas - foi confirmado em uma reunião dos organizadores sobre a égide da Society of Arts, presidida pelo Príncipe Albert desde $1843 .{ }^{4}$ No que concerne a organização da exposição, uma comissão real 'that would have the authority of a state institution [...] but would also be a body of independent of state finances an untainted by by political connections' (DAVIS 1999: 27), foi formada para assumir a sua direção, também sendo presidida pelo Príncipe Albert. Apesar do fato da participação de muitos indivíduos ter sido crucial para o desenvolvimento dos planos para a exposição é a figura de Albert e Cole que se destaca quando se fala sobre os principais responsáveis para o sucesso do empreendimento:

When Henry Cole and Prince Albert set in motion plans for the 1851 exhibition, they were building on the combined foundations of the Industrial Revolution, the scope and reserves of the British Empire and the entire intellectual and social outlook of the period. Although they might not have realised it, they were also giving a new shape to a universal human attribute - they were making an exhibition of themselves or, more simply, showing-off. It is a trait much despised when found in the individual but the life-blood of commerce and industry. (ALLWOOD 2001: 1)

Em outras palavras, eles parecem ter antecipado o papel de celebridades assumido por muitos curadores contemporâneos na mesma medida em que promoviam transformações profundas no âmbito das exposições. Talvez por causa desse ímpeto exibicionista esses eventos também buscavam se tornar sempre maiores do que os seus antecessores. No entanto, como afirma Allwood: 'London in 1862 had far more, and in many cases far better exhibits than the 1851 exhibition, but today who knows that there was an International Exhibition in London in 1862, let alone where it was or what the building looked like?' (2001: 1) 


\section{À guisa de conclusão}

A Galeria de Pinturas foi o principal destaque da Exposição Internacional de 1862, bem como o eixo ao redor do qual se articulou a análise aqui apresentada. Ainda em comparação com o Palácio de Cristal, vale observar o fato de que um dos motivos da não inclusão de pinturas na exposição inaugural de 1851, para além das limitações técnicas do edifício, teria sido a concomitância com o salão da Royal Academy (Bryant 2014: 60). Este aspecto deve ser ressaltado, uma vez que isso indicaria que a internacionalidade pretendida pela exposição de 1851 não teria se estendido para o campo das artes visuais. No entanto, o próprio Bryant refuta a hipótese de que as artes visuais tenham sido subestimadas para o Império britânico:

\footnotetext{
Fine art was not a novel addition in 1862, simply assembled in reply to its presence in Paris in 1855: it featured in the Great Exhibition of 1851, despite many historians' accounts to the contrary. The British were not totally preoccupied with industry, trade and empire (Bryant 2014: 60).
}

Apesar dessa controvérsia, somente em 1862 a exposição de Londres tornar-se plenamente internacional, com a inclusão de uma galeria de pinturas. $O$ plano era para que o edifício a ser construído para a exposição permanecesse para receber a coleção da National Gallery que ainda compartilhava um espaço provisório com a Royal Academy (Bryant 2014: 61). Um artigo se refere ao edifício como um

ambitious project of raising at Brompton a revival of the Alexandrian "Museum" out of the débris of the 1851 Exhibition. The removal of the National Gallery from Trafalgar Square, which formed an element in the calculation, happened to arrest public attention when other proposals would not have possessed an interest outside learned circles. (INTERNATIONAL EXHIBITION 1862c: 181)

Do ponto de vista do caráter internacional da exposição, chama a atenção a afirmação de uma superioridade da representação britânica. Se por um lado, a mostra internacional de artes era promovida como um âmbito de representação das diferenças entre a arte dos diversos países participantes, por outro lado, a afirmação da galeria dedicada às pinturas do Reino Unido como o principal destaque da exposição demonstra a clara estratégia de construção de hierarquias e hegemonias no campo das artes. Apesar da abrangência da seleção incluir 
productions of modern Art, gathered out of the museums, the palaces, the private galleries, and the studios of almost every European capital', a crítica enfatiza uma perspectiva nacionalista na qual pode-se 'trace the progress and see the lates results of English Art, side by side with the art of every other contemporary school.' (INTERNATIONAL EXHIBITION 1862e: 246).

Para Bryant, se por um lado essas exposições levantavam

\begin{abstract}
the state of modern art around the world', elas 'also provided the opportunity for nations to present their national identities through the histories of their own art. For the host nation, the Exhibition was a landmark in the definition, presentation and appreciation of the British School of painting and sculpture (BRYANT 2014: 59).
\end{abstract}

A historiografia sobre as megaexposições internacionais tem abordado as questões da definição de identidades nacionais no contexto de expansão do capitalismo do século XIX e do estabelecimento de trocas comerciais em escala global. Muito embora se fale dos desenvolvimentos do processo de colonização no auge de um sistema Imperial de dominação política e econômica, as leituras se voltam para a forma como cada país em particular definiu no âmbito das exposições uma representação de suas culturas e identidades nacionais. No limite, essas reflexões permitem compreender a forma países centrais como a França e o Reino Unido, bem como países periféricos articulavam suas representações dentro de um sistema binário de oposições entre o nacional e o internacional. Partindo assim de uma reflexão sobre a origem das megaexposições internacionais de arte contemporânea, poder-se-ia ir além nessas reflexões sobre os nacionalismos nas artes, adentrando em reflexões sobre transculturalidade no contexto atual de globalização cultural.

Assim, o recuo histórico realizado pela pesquisa abre caminho para futuras análises, como por exemplo, sobre a questão da recepção da arte brasileira no Reino Unido e sua consequente internacionalização, tema a ser tratado em outro artigo. Por hora, chegamos à conclusão de que as exposições foram historicamente locais privilegiados para trocas culturais. Não somente as exposições internacionais, mas todo um sistema de representação, ou, dito em outras palavras, de um aparato colonial que contribuiu no estabelecimento de identidades nacionais na esfera internacional das exposições. Por meio desse sistema, os impérios buscaram inscrever uma determinada construção de sua própria cultura dentro de uma perspectiva internacional. Entretanto, apesar do fato das exposições do século XIX ofereceram à 'todas as nações' o acesso à uma linguagem universal, o universalismo de uma representação dita internacional não era reconhecido 
em todos os países. Nesse sentido, as exposições internacionais poderiam sim ser vistas como uma grande 'babel', contrariando a crítica da época, onde muitas línguas diferentes são faladas, representando suas respectivas nacionalidades, sobretudo no campo das artes. Por outro lado, buscava-se ao mesmo tempo falar a 'língua franca' do internacionalismo, alinhada ideologicamente ao livre comércio e ao capitalismo do século XIX. Não à toa no âmbito dos debates sobre o pós-colonial, as exposições de arte têm se transformado em um objeto de estudo bastante revisitado, bem como as próprias megaexposições internacionais de arte contemporânea transformaram-se em plataformas de debate e discussão sobre o tema.

1 Preziosi escreve a partir da perspectiva de uma revisão crítica da história da arte que aconteceu nas últimas décadas do século XX. No seu ponto de vista, a história da arte e a museologia foram constituídas enquanto disciplinas acadêmicas no centro de um sistema mais amplo de estabelecimento de uma hegemonia cultural pelos países ditos 'ocidentais'. Dessa forma, ele associa o 'universalismo' proposto por essas disciplinas modernas à definição de uma identidade 'ocidental' e 'civilizada' que definiria ao mesmo tempo o 'outro', ou seja, tudo aquilo que não é 'ocidental', como 'primitivo' e 'exótico', na melhor das alternativas, ou 'bárbaro' no limite.

2 Talvez isso se deu pelo fato da representação do Império do Brasil em 1862 ter suprimido o seu 'sotaque', diferentemente das representações que ocorreram nas Expositions Universelles de Paris e nas World Fairs dos Estados Unidos analisadas por Rezende (2010). Segundo ela, o Império do Brasil foi representado de maneira informal na Grande Exposição de 1851 e na Exposição Universal de 1855, perdendo assim uma oportunidade de se apresentar nesse âmbito como uma nação 'civilizada' (REZENDE, 2010: 16-17). A despeito disso, da Exposição Internacional de 1862 até o final do Império em 1889, as exposições 'became prime sites to form symbols and connections for national unity and identity, at home and abroad', afirma Rezende. Sua Tese apresenta 'a particular notion of nationality, a notion that aimed to identify Brazil as a distinct nation abroad and as a cohesive unit for foreigners and its people' que foram materializadas por meio dos objetos e materiais exibidos (REZENDE, 2010: 18). A autora foca também no papel desempenhado pelas elites e representantes governamentais na organização dessas representações de acordo com suas visões políticas, por meio da construção de uma noção de 'brasilidade'

3 Um compêndio que abarca um vasto período de tempo, de meados do século XIX até a Segunda Guerra Mundial, ou seja, do surgimento ao ocaso de uma série de mega exposições como 'Word's Fairs', 'Expositions Universelles', e 'Weltausstellungen'.

4 Nesse primeiro encontro participaram Scott Russel, secretário da Society of Art, Francis Fuller, agente imobiliário de Londres e Thomas Cubitt, assessor para a construção do edifício para abrigar o evento, e sócio de seu irmão William, um dos membros da Royal Commission juntamente com o arquiteto C. R. Cockeferell do Ashmolean Museum, entre outros. Dentre os membros do merece destaque o Primeiro Ministro, Lord John Russell. 


\section{Referências}

ALLWOOD, John. The Great Exhibitions: 150 years. 2 ed. London: Exhibition Consultants Ltd., 2001.

ALTICK, Richard. The Shows of London. Cambridge, Mass.: Belknap Press, 1978.

BATAILLE, George; Michelson, Annette, Museum, October, Vol. 36, Georges Bataille: Writings on Laughter, Sacrifice, Nietzsche, Unknowing, 1986, pp. 24.

BENNETT, Tony. The Birth of the Museum : History, Theory, Politics. Culture : Policies and Politics. London: Routledge, 1995.

BRYANT, Julius. The progress and present condition of Modern Art': Fine Art at the 1862 Exhibition. The Journal of the Decorative Arts Society 1850 - the Present, No. 38, Almost Forgotten: the International Exhibtion of 1862, 2014, pp. 58-81.

DAVIS, John R. The Great Exhibition. Stroud: Sutton, 1999.

DISHON, Dale. South Kensington's Forgotten Palace: The Rise and Fall of the 1862 Exhibition. The Journal of the Decorative Arts Society 1850 - the Present, No. 38, Almost Forgotten: the International Exhibtion of 1862, 2014, pp. 20-43.

DUNCAN, Carol. From the Princely Gallery to the Public Art Museum: The Louvre Museum and the National Gallery, London. In Preziosi, Donald; Farago, Claire. Grasping the World : The Idea of the Museum. Aldershot, Hants, England: Ashgate Pub, 2004, p.250-277.

FILIPOVIC, Elena; VAN HAL; Marieka, ØVSTEB Ø, Sølveig. The Biennial Reader: Anthology of essays on the global phenomena of biennials. Ostfildern: Hatje Cantz, 2010.

GREENHALGH, Paul. Ephemeral vistas: the expositions universelles, great exhibitions and world's fairs, 1951-1939. Manchester University Press, 1988.

HUTCHISON, Sidney C. The History of the Royal Academy 1768-1986. 2 ed. London: Royce, 1986.

INTERNATIONAL EXHIBITION. Remarks on the Foreign Pictures Now in London, 1862a, p.50-57.

INTERNATIONAL EXHIBITION. Pictures, British and Foreign. Edinburgh: Blackwood, 1862b, p.353-371.

MOREIRA, Francisco. Relatório Sobre a Exposição Internacional em 1862 Apresentado a S. M. o Imperador, Thomas London: Brettell, 1863.

PEVSNER, Nikolaus. High Victorian Design: A Study of the Exhibits of 1851. London: Architectural Press, 1951.

PREZIOSI, Donald. Brain of the Earth's Body : Art, Museums, and the Phantasms of Modernity. Minneapolis: University of Minnesota Press, 2003.

REZENDE, Lívia. The Raw and the Manufactured: Brazilian Modernity and National Identity as Projected in International Exhibitions (1862-1922), PhD thesis, Royal College of Art, 2010. YOUNG, Paul. Globalization and the Great Exhibition : The Victorian New World Order. Palgrave Studies in Nineteenth-Century Writing and Culture. New York: Palgrave Macmillan, 2009. 